\title{
Cell Gas Free Energy as an Approximation of the Continuous Model
}

\author{
Vira A. Boluh1, Alexei L. Rebenko² \\ ${ }^{1}$ Faculty of Mathematics, Zhytomyr, Ukraine \\ ${ }^{2}$ Institute of Mathematics, Ukrainian National Academy of Sciences, Kyiv, Ukraine \\ Email: virashevchuk@ukr.net, rebenko@imath.kiev.ua
}

Received 1 February 2015; accepted 15 February 2015; published 25 February 2015

Copyright (C) 2015 by authors and Scientific Research Publishing Inc.

This work is licensed under the Creative Commons Attribution International License (CC BY). http://creativecommons.org/licenses/by/4.0/

(c) (†) Open Access

\begin{abstract}
A continuous infinite system of point particles interacting via two-body strong superstable potential is considered in the framework of cell gas (CG) model of classical statistical mechanics. We consider free energy of this model as an approximation of the correspondent value of the continuous system. It converges to the free energy of the conventional continuous gas if the parameter of approximation $a \rightarrow 0$ for any values of an inverse temperature $\beta>0$ and volume per particle $v>0$.
\end{abstract}

\section{Keywords}

\section{Strong Superstable Potential, Quasi-Lattice Approximation, Cell Gas}

\section{Introduction}

One of the most important mathematical problem of statistical mechanics is description of the gas-liquid phase transition within the framework of standard model of 2-partical Lenarda-Johnson type intermolecular interaction. The presence of phase transition at some temperature $T_{c}=1 / k \beta_{c}$ ( $k$ is the Boltzmann constant, $\beta$ is inverse temperature in units of inverse energy) means that at $\beta=\beta_{c}$ in some interval of change of density $\rho$, pressure $p$ does not depend on density or a specific volume of $v=1 / \rho$ (see, for example [1]). Taking into account the well-known thermodynamics formulas it means that free energy of the system depends on specific volume $v$ linearly in the indicated interval of change of density. This result was obtained as early as the end of 60th for the lattice gas model in the articles of F. A. Berezin and Ya. G. Sinai [2] for unpositive potentials and R. L. Dobrushin [3] for more general potentials of interaction.

However, the lattice gas is some kind of "toy" model which is very far from the real continuous system. The model of cell-type gas, which actually is the model of the continuous system of point particles and differs from 
the standard model of gas only by determination of the phase (configuration) space, was offered in recent work [4] of one of the authors of this article.

Cell gas in $\mathbb{R}^{d}$ is a continuous gas but its space of configurations is arranged so that for a given partition $\overline{\Delta_{a}}$ of $\mathbb{R}^{d}$ into elementary hiper-cubes $\Delta \subset \mathbb{R}^{d}$ with a rib $a>0$ there is no more then one point particle in each cell (cube). These particles move in $\mathbb{R}^{d}$ and interact via two-body strong superstable potential $\phi$. According to the results of articles [5] [6] and [7] the correlation functions and the pressure of cell gas system tend to the corresponding values of conventional continuous gas at $a \rightarrow 0$. Within the framework of the grand canonical ensemble this result followed from a convenient representation of the corresponding quantities by Poisson integrals on the configuration space of the system. In this short paper we establish a similar result for the free energy of the system. This result requires more hard work as the corresponding representation in the canonical ensemble less convenient for mathematical calculations.

Why do we need this result? In the article [4] it was shown that it was possible to introduce an approximation of the interaction potential in such a way that the cell gas model grows into the model of the lattice gas on the lattice $a \mathbb{Z}^{d}$, and at $a \rightarrow 0$ both models coincide with a model which describes the continuous statistical system. Therefore we consider the result of this article as the first modest step to realization of the Dobrushin's way [3] to solve the phase transition problem in continuum.

\section{Notations and Main Results}

\subsection{Configuration Space}

Let $\mathbb{R}^{d}$ be a $d$-dimensional Euclidean space. The set of positions $\left\{x_{i}\right\}_{i \in N}$ of identical point particles is considered to be a locally finite subset in $\mathbb{R}^{d}$ and the set of all such subsets creates the configuration space:

$$
\Gamma=\Gamma_{\mathbb{R}^{d}}:=\left\{\gamma \subset \mathbb{R}^{d}|| \gamma \cap \Lambda \mid<\infty, \text { for all } \Lambda \in \mathcal{B}_{c}\left(\mathbb{R}^{d}\right)\right\}
$$

where $|A|$ denotes the cardinality of the set $A$ and $\mathcal{B}_{c}\left(\mathbb{R}^{d}\right)$ denote the systems of all bounded Borel sets in $\mathbb{R}^{d}$. We also need to define the space of finite configurations $\Gamma_{0}$ :

$$
\Gamma_{0}=\coprod_{n \in N_{0}} \Gamma^{(n)}, \quad \Gamma^{(n)}:=\left\{\eta \subset \mathbb{R}^{d}|| \eta \mid=n\right\}, \quad N_{0}=N \cup\{0\}
$$

and $\Gamma_{\Lambda}$ :

$$
\Gamma_{\Lambda}:=\{\gamma \in \Gamma \mid \gamma \subset \Lambda\}
$$

By $\mathcal{B}\left(\Gamma_{\Lambda}\right)$ we denote the corresponding $\sigma$-algebra on $\Gamma_{\Lambda}$. For the given intensity measure $\sigma$ (in this context $\sigma$ is Lebesgue measure on $\mathcal{B}\left(\mathbb{R}^{d}\right)$ ) and any $n \in N$ the product measure $\sigma^{\otimes n}$ can be considered as a measure on

$$
\overline{\left(\mathbb{R}^{d}\right)^{n}}=\left\{\left(x_{1}, \cdots, x_{n}\right) \in\left(\mathbb{R}^{d}\right)^{n} \mid x_{k} \neq x_{l} \text { if } k \neq l\right\}
$$

and hence as a measure $\sigma^{(n)}$ on $\Gamma^{(n)}$ through the map

$$
\operatorname{sym}_{n}: \overline{\left(\mathbb{R}^{d}\right)^{n}} \ni\left(x_{1}, \cdots, x_{n}\right) \mapsto\left\{x_{1}, \cdots, x_{n}\right\} \in \Gamma^{(n)}
$$

Define the Lebesgue-Poisson measure $\lambda_{\sigma}$ on $\mathcal{B}\left(\Gamma_{0}\right)$ by the formula:

$$
\lambda_{\sigma}:=\sum_{n \geq 0} \frac{1}{n !} \sigma^{(n)}
$$

The restriction of $\lambda_{\sigma}$ to $B\left(\Gamma_{\Lambda}\right)$ we also denote by $\lambda_{\sigma}$. For more detailed structure of the configuration spaces $\Gamma, \Gamma_{0}, \Gamma_{\Lambda}$ and measures on them see e.g. [8] (see also latest review [9]).

Let $a>0$ be arbitrary. Following [10] for each $r \in a \mathbb{Z}^{d}$ we define an elementary cube with an edge $a$ and a center $r$ 


$$
\Delta_{a}(r):=\left\{x \in \mathbb{R}^{d} \mid\left(r^{i}-a / 2\right) \leq x^{i}<\left(r^{i}+a / 2\right)\right\}
$$

We will write $\Delta$ instead of $\Delta_{a}(r)$, if a cube $\Delta$ is considered to be arbitrary and there is no reason to emphasize that it is centered at the concrete point $r \in a \mathbb{Z}^{d}$. Let $\bar{\Delta}_{a}$ be the partition of $\mathbb{R}^{d}$ into cubes $\Delta_{a}(r)$. Without loss of generality we consider $\Lambda$ in the form of a large cube and only that $\Lambda \in \mathcal{B}_{c}\left(\mathbb{R}^{d}\right)$ and subsets $X, Y \subset \Lambda$ which are union of cubes $\Delta_{a}(r)$ and corresponding partition:

$$
\bar{\Delta}_{a, Y}:=\bar{\Delta}_{a} \cap Y, \quad Y \in\{X, \Lambda \backslash X\}
$$

Then for any $X \subseteq \Lambda$ which is a union of cubes $\Delta \in \bar{\Delta}_{a}$ define

$$
\Gamma_{X}^{\text {dil }}:=\left\{\gamma \in \Gamma_{X} \mid N_{\Delta}(\gamma)=0 \vee 1 \text { for all } \Delta \subset X\right\}
$$

and

$$
\Gamma_{X}^{\text {den }}:=\left\{\gamma \in \Gamma_{X} \mid N_{\Delta}(\gamma) \geq 2 \text { for all } \Delta \subset X\right\}
$$

Definition 2.1. Infinite system of point particles in $\mathbb{R}^{d}$ with given partition $\bar{\Delta}_{a}$ and configuration space $\Gamma^{\mathrm{dil}}:=\Gamma_{\mathbb{R}^{d}}^{\mathrm{dil}}$ is called cell gas system of particles.

For detail structure of this model see [4].

\subsection{Definition of the System}

We consider a general type of two-body interaction potential $V_{2}(x, y)=\phi(|x-y|)$, where $\phi: \mathbb{R}_{+} \rightarrow \mathbb{R} \cup\{+\infty\}$ satisfies the following properties.

(A): Assumption on the interaction potential. Potential $\phi$ is continuous on $\mathbb{R}_{+} \backslash\{0\}$ and there exist $r_{0}>0, R>r_{0}, \varphi_{0}>0, \varphi_{1}>0$, and $\varepsilon_{0}>0$ such that:

$$
\begin{aligned}
& \text { 1) } \phi(|x|) \equiv-\phi^{-}(|x|) \geq-\frac{\varphi_{1}}{|x|^{d+\varepsilon_{0}}} \text { for }|x| \geq R \\
& \text { 2) } \phi(|x|) \equiv \phi^{+}(|x|) \geq \frac{\varphi_{0}}{|x|^{s}}, s \geq d \text { for }|x| \leq r_{0}
\end{aligned}
$$

where

$$
\phi^{+}(|x|):=\max \{0, \phi(|x|)\}, \quad \phi^{-}(|x|):=-\min \{0, \phi(|x|)\}
$$

The potentials of this type are strong superstable.

Definition 2.2. Interaction is called strong superstable (SSS), if there exist $a_{0}>0$, and constants

$A(a)>0, B(a) \geq 0$, and $m \geq 2$ such that for any $0<a \leq a_{0}$ and any $\gamma \in \Gamma_{0}$ an interaction energy of particles satisfy the following inequality:

$$
U(\gamma) \geq A(a) \sum_{\Delta \in \bar{\Delta}_{a}:\left|\gamma_{\Delta}\right| \geq 2}\left|\gamma_{\Delta}\right|^{m}-B(a)|\gamma|
$$

Remark 2.1. Superstable interactions were introduced by D. Ruelle (see [11] or [12], Ch. 3.2.9 and [10]). Y. M. Park (see [14]) was the first, who used the condition (12) with $m>2$ for the proof of bounds for exponent of local number operator of quantum systems of interacting Bose gas. We have changed the definition of strong superstability including the case $m=2$, but with the constants which depends on parameter a (see, e.g., [13] [4]). SSS potentials include all interaction potentials which are nonintegrable in the initial point.

One of the most popular example which is used in molecular physics is Lenard-Jonson potential:

$$
\phi(|x|)=\frac{C}{|x|^{12}}-\frac{D}{|x|^{6}}
$$

where constants $C>0, D>0$. In this article we consider the potentials of this type. The typical behavior of such potentials is shown in Figure 1. 


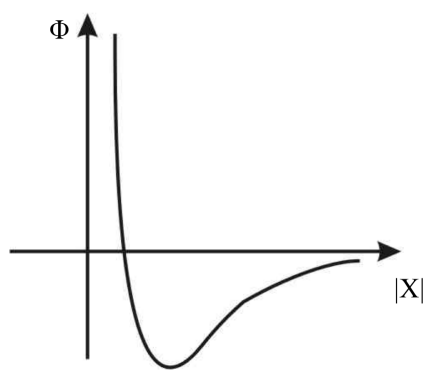

Figure 1. The typical behavior of the potentials.

Remark 2.2. For the potentials which are considered in this article (see (9)-(11)) the corresponding constants $A(a)$ and $B(a)$ have the following form:

$$
A(a)=C_{s, d}-\frac{v_{0}}{2^{s / d}}, \quad B(a)=\frac{v_{0}}{2}, \quad m=1+\frac{s}{d}, \quad C_{s, d}=\frac{1}{2^{2 s+1}}\left(\frac{2 \pi^{\frac{d}{2}}}{d \Gamma\left(\frac{d}{2}\right)}\right)^{\frac{s}{d}} \frac{\varphi_{0}}{a^{s}}
$$

where

$$
v_{0}(a):=\sum_{\Delta \in \bar{\Delta}} \sup _{x \in \Delta} \phi^{-}(|x|)
$$

See for the proof [13].

\subsection{Partition Functions, Free Energy and Pressure}

The main physical characteristics of the system are determined by thermodynamic potentials that associated with small and grand partition functions by the following formulas: 1) free energy

$$
f(v, \beta)=\lim _{\substack{N \rightarrow \infty \\ \Lambda \uparrow \mathbb{R}^{d}}} f_{\Lambda}(N, \beta):=\lim _{\substack{N \rightarrow \infty \\ \Lambda \uparrow \mathbb{R}^{d}}} \frac{1}{N} \log Z_{\Lambda}(N, \beta)
$$

where limit is done in such a way that volume per particle $\sigma(\Lambda) / N \rightarrow v, \beta=1 / k T$, and small partition function

$$
\begin{aligned}
& Z_{\Lambda}(N, \beta)=\int_{\Gamma_{\Lambda}^{(N)}} \mathrm{e}^{-\beta U(\eta)} \lambda_{\sigma}(\mathrm{d} \eta) \\
& U(\eta)=U_{\phi}(\eta):=\sum_{\{x, y\} \subset \eta} \phi(|x-y|), \quad \eta \in \Gamma_{\Lambda}^{(N)}
\end{aligned}
$$

2) pressure

$$
p(z, \beta)=\lim _{\Lambda \uparrow \mathbb{R}^{d}} p_{\Lambda}(z, \beta)=\lim _{\Lambda \uparrow \mathbb{R}^{d}} \frac{k T}{\sigma(\Lambda)} \log Z_{\Lambda}(z, \beta)
$$

where $z$ is activity of the system and

$$
Z_{\Lambda}(z, \beta)=\int_{\Gamma_{\Lambda}} \mathrm{e}^{-\beta U(\gamma)} \lambda_{z \sigma}(\mathrm{d} \gamma)
$$

The correspondent values for cell gas model are defined by the same formulas but with help of partition functions(see Definition 2.1):

$$
Z_{\Lambda}^{(a)}(z, \beta)=\int_{\Gamma_{\Lambda}^{(\mathrm{dil})}} \mathrm{e}^{-\beta U(\gamma)} \lambda_{z \sigma}(\mathrm{d} \gamma)=\int_{\Gamma_{\Lambda}} \prod_{\Delta \in \bar{\Delta}_{a, \Lambda}} \chi_{-}^{\Delta}(\gamma) \mathrm{e}^{-\beta U(\gamma)} \lambda_{z \sigma}(\mathrm{d} \gamma)
$$

and 


$$
Z_{\Lambda}^{(a)}(z, \beta)=\int_{\Gamma_{\Lambda}^{(N)}} \prod_{\Delta \in \bar{\Delta}_{a, \Lambda}} \chi_{-}^{\Delta}(\gamma) \mathrm{e}^{-\beta U(\gamma)} \lambda_{\sigma}(\mathrm{d} \gamma)
$$

where

$$
\chi_{-}^{\Delta}(\gamma)=\chi_{-}^{\Delta}\left(\gamma_{\Delta}\right)= \begin{cases}1, & \text { for } \gamma \text { with }\left|\gamma_{\Delta}\right|=0 \vee 1 \\ 0, & \text { otherwise }\end{cases}
$$

Remark 2.3. The product of functions $\chi_{-}^{\Delta}$ in definition of statistical sums $Z_{\Lambda}^{(a)}(z, \beta)$ and $Z_{\Lambda}^{(a)}(N, \beta)$ limits configuration space of the system of point particles to the $\Gamma_{\Lambda}^{\text {(dil) }}$ (see def. (2.7)). However, the system is continuous as particles are arranged in all points of the space $\mathbb{R}^{d}$, but at the same time their joint position are defined only in $\Gamma^{\text {(dil) }} \subset \Gamma$.

Now, we can formulate the main result of the paper.

Theorem 1 Suppose that the interaction potential $\phi$ satisfies the assumptions A (see (9), (10)). Then there exists some $0 \leq v_{0}<\infty$, such that for all $v>v_{0}$ there exist the limit

$$
f^{(a)}(v, \beta)=\lim _{\substack{N \rightarrow \infty \\ \Lambda \uparrow \mathbb{R}^{d}}} f_{\Lambda}^{(a)}(N, \beta):=\lim _{\substack{N \rightarrow \infty \\ \Lambda \uparrow \mathbb{R}^{d}}} \frac{1}{N} \log Z_{\Lambda}^{(a)}(N, \beta)
$$

for any $v>v_{0}$. The function $f^{(a)}(v, \beta)$ is monotone nondecreasing concave continuous function of $v$.

Theorem 2 Suppose that the interaction potential $\phi$ satisfies the assumptions A (see (9), (10)). Then for any $\varepsilon>0$ there exists $a_{1}=a_{1}(v, \varepsilon)>0$ such that:

$$
\left|f(v, \beta)-f^{(a)}(v, \beta)\right|<\varepsilon
$$

holds for all positive $v, \beta$ and $a \in\left(0, a_{1}(v, \varepsilon)\right)$.

\section{The Proof of the Main Results}

The proof of the Theorem 2.1 is the same as the corresponding proof of such theorem for $f(v, \beta)$ in [15]. The only remark to the proof is that the construction of auxiliary partitions into cubes in [15] should be agreed with the partition $\bar{\Delta}_{a}$.

To prove the Theorem 2.2 we insert the unite

$$
1=\prod_{\Delta \in \bar{\Delta}_{a, \Lambda}}\left[\chi_{-}^{\Delta}(\gamma)+\chi_{+}^{\Delta}(\gamma)\right]=\sum_{\bar{X} \subseteq \bar{\Delta}_{a, \Lambda}} \tilde{\chi}_{+}^{\bar{X}}(\gamma) \tilde{\chi}_{-}^{\bar{\Delta}_{a, \Lambda} \backslash \bar{X}}(\gamma)
$$

where $\chi_{+}^{\Delta}(\gamma)=1-\chi_{-}^{\Delta}(\gamma)$

$$
\begin{aligned}
& \tilde{\chi}_{ \pm}^{\bar{Y}}(\gamma):=\prod_{\Delta \in \bar{\Delta}_{a, Y}} \chi_{ \pm}^{\Delta}(\gamma) \\
& \sum_{\bar{X} \subseteq \bar{\Delta}_{a, \Lambda}}(\cdots)=\sum_{k=0}^{N_{\Lambda}} \sum_{\bar{x}_{k} \subseteq \bar{\Delta}_{a, \Lambda}}(\cdots)
\end{aligned}
$$

and $\bar{X}_{k}:=\left\{\Delta_{1}, \cdots, \Delta_{k}\right\}, \quad N_{\Lambda}:=\sigma(\Lambda) / a^{d}$, into the expression (18) for small partition function. Then

$$
Z_{\Lambda}(N, \beta)=\sum_{\bar{X} \subseteq \bar{\Delta}_{a, \Lambda}} \int_{\Gamma_{\Lambda}^{(N)}} \tilde{\chi}_{+}^{\bar{X}}(\gamma) \tilde{\chi}_{-}^{\bar{a}_{a, \Lambda} \backslash \bar{X}}(\gamma) \mathrm{e}^{-\beta U(\gamma)} \lambda_{\sigma}(\mathrm{d} \gamma)
$$
form:

Separating the first term of the expansion which corresponds to the value $\bar{X}=\varnothing$ we can rewrite (30) in the

where

$$
Z_{\Lambda}(N, \beta)=Z_{\Lambda}^{(a)}(N, \beta) Z_{\Lambda}^{(+)}(N, a, \beta)
$$

$$
Z_{\Lambda}^{(+)}(N, a, \beta)=1+\frac{1}{Z_{\Lambda}^{(a)}(N, \beta)} \sum_{\varnothing \neq \bar{X} \subseteq \bar{\Delta}_{a, \Lambda}} \int_{\Gamma_{\Lambda}^{(N)}} \tilde{\chi}_{+}^{\bar{X}}(\gamma) \tilde{\chi}_{-}^{\overline{\bar{A}}_{a, \Lambda} \backslash \bar{X}}(\gamma) \mathrm{e}^{-\beta U(\gamma)} \lambda_{\sigma}(\mathrm{d} \gamma)
$$

The Equation (31) gives: 


$$
\begin{gathered}
f_{\Lambda}(N, \beta)=f_{\Lambda}^{(a)}(N, \beta)+\Delta f_{\Lambda}^{(a)}(N, \beta) \\
\Delta f_{\Lambda}^{(a)}(N, \beta):=\frac{1}{N} \log Z_{\Lambda}^{(+)}(N, a, \beta)
\end{gathered}
$$

To estimate the second term in (33) we split the energy $U(\gamma)$ in every term of the sum in (32):

$$
U(\gamma)=U\left(\gamma_{X}\right)+W\left(\gamma_{X} ; \gamma_{\Lambda \backslash X}\right)+U\left(\gamma_{\Lambda \backslash X}\right)
$$

where

$$
W(\gamma ; \eta):=\sum_{\substack{x \in \gamma \\ y \in \eta}} \phi(|x-y|), \quad \gamma, \eta \in \Gamma_{0}
$$

and use SSS inequality (12). Then

$$
\mathrm{e}^{-\beta U\left(\gamma_{X}\right)} \mathrm{e}^{-\beta W\left(\gamma_{X} ; \gamma_{\Lambda \backslash X}\right)} \leq \prod_{\Delta \in \bar{\Delta}_{a, X}} \mathrm{e}^{-\beta A(a)\left|\gamma_{\Delta}\right|^{m}+\beta C(a)\left|\gamma_{\Delta}\right|}:=E_{X}
$$

where

$$
C(a)=B(a)+v_{0}(a)
$$

We denote the integral in (32) (after estimating (37)) by the letter $I_{X}$ and rewrite an expression for $I_{X}^{\prime}=I_{X} N$ ! in the following form

$$
I_{X}^{\prime}=\left(\int_{X} \mathrm{~d} x_{1}+\int_{\Lambda \backslash X} \mathrm{~d} x_{1}\right) \cdots\left(\int_{X} \mathrm{~d} x_{N}+\int_{\Lambda \backslash X} \mathrm{~d} x_{N}\right) E_{X} \tilde{\chi}_{+}^{\bar{X}}\left(\gamma_{X}\right) \mathrm{e}^{-\beta U\left(\gamma_{\Lambda \backslash X}\right)} \tilde{\chi}_{-}^{\bar{\Delta}_{a, \Lambda} \backslash \bar{X}}\left(\gamma_{\Lambda \backslash X}\right)
$$

Every set in $\bar{X}$ is an union of $k$ cubes $\Delta_{1}, \cdots, \Delta_{k}, k \in\left\{1, \cdots, N_{\Lambda}\right\}$. There are at least two variables from the configuration $\gamma=\left\{x_{1}, \cdots, x_{N}\right\}$ in every cube $\Delta_{j}, j \in\{1, \cdots, k\}$. Denote the number of variables that are in cubes $\Delta_{1}, \cdots, \Delta_{k}$ by the letters $M=m_{1}+\cdots+m_{k}$. It is clear that $M \in\{2 k, \cdots, N\}$ and $m_{j} \geq 2, j \in\{1, \cdots, k\}$. Among all $2^{N}$ terms which appear in the right side of (39)) does not vanish only those terms in which the integration is performed with respect to the variables $\left\{x_{1}, \cdots, x_{M}\right\}$ over region $X_{k}=\bigcup_{i=1}^{k} \Delta_{i}$ and with respect to the variables $\left\{x_{M+1}, \cdots, x_{N}\right\}$ over region $\Lambda \backslash X_{k}=\bigcup_{i=k+1}^{N} \Delta_{i}$. Due to the symmetry of the integrand with respect to permutations of variables $\left\{x_{1}, \cdots, x_{N}\right\}$ the number of terms in $I_{X}$ which correspond to a fixed $M$ is $N ! /(N-M) ! M !$. In the same way every integral over $X_{k}$ one can represent as a sum of integrals over cubes $\Delta_{1}, \cdots, \Delta_{k}$. Next, we take into account that the variables $\left\{x_{1}, \cdots, x_{M}\right\}$ can be placed into $k$ cubes so that each cube $\Delta_{j}$ has exactly $m_{j}$ variables by $M ! /\left(m_{1} ! \cdots m_{k} !\right)$ ways. As a result we have:

$$
Z_{\Lambda}^{(+)}(N, a, \beta) \leq 1+\sum_{k=1}^{N_{\Lambda}} \sum_{\left\{\Lambda_{1}, \cdots, \Delta_{k}\right\} \subset \bar{\Delta}_{a, \Lambda}} \sum_{M=2 k}^{N} a^{d M} \mathrm{e}^{\beta C(a) M} \sum_{\substack{m_{1}, \cdots, m_{k}: m_{j} \geq 2 \\ m_{1} \cdots+\cdots, m_{k}=M}}\left(\prod_{j=1}^{k} \frac{\mathrm{e}^{-\beta A(a) m_{j}^{m}}}{m_{j} !}\right) \frac{Z_{\Lambda \backslash X_{k}}^{(a)}(N-M, \beta)}{Z_{\Lambda}^{(a)}(N, \beta)}
$$

To estimate the ratio of the partition functions in (40) we use the following lemma.

Lemma 1 Suppose that the interaction potential $\phi$ satisfies the assumptions A (see (9), (10)). Then there exists constant $K>0$ such that

$$
\frac{Z_{\Lambda \backslash X_{k}}^{(a)}(N-M, \beta)}{Z_{\Lambda}^{(a)}(N, \beta)} \leq K^{M}
$$

for any $\beta>0, v>0$ and sufficiently large cube $\Lambda$.

Proof. Let us fix some $\bar{v}>0$ and sufficiently large cube $\Lambda$ in such a way that $\sigma(\Lambda) / N \geq \bar{v}$. Following Dobrushin and Minlos [16] we introduce an auxiliary potential

$$
\phi_{\bar{a}}(|x|)= \begin{cases}0, & \text { for }|x| \leq \bar{a} \\ \phi(|x|), & \text { for }|x|>\bar{a}\end{cases}
$$

with any $a<\bar{a}<r_{0}$ (see (10)). The proof of the lemma follows from the estimate of ratio of configuration integral $Q^{(a)}(N, \Lambda, \beta)=N ! Z_{\Lambda}^{(a)}(N, \beta)$ (see also [16], Lemma $3^{\prime}$ ): 


$$
\frac{Q^{(a)}(N+1, \Lambda, \beta)}{Q^{(a)}(N, \Lambda, \beta)} \geq k \sigma(\Lambda)
$$

with $k=k(\bar{a}, \bar{v})$. To prove (43) write $Q^{(a)}(N+1, \Lambda, \beta)$ in the following form:

$$
Q^{(a)}(N+1, \Lambda, \beta)=\int_{\Gamma_{\Lambda}^{(N)}} \mathrm{e}^{-\beta U(\gamma)} \mathrm{d} \gamma \int_{\Lambda} \mathrm{d} x \mathrm{e}^{-\beta W(x ; \gamma)} \prod_{\Delta \in \bar{\Delta}_{a, \Lambda}} \chi_{-}^{\Delta}(\gamma \cup\{x\})
$$

where $\gamma=\left\{x_{1}, \cdots, x_{N}\right\}, \mathrm{d} \gamma=\mathrm{d} x_{1} \cdots \mathrm{d} x_{N}$. Define the region

$$
\tilde{\Lambda}_{\bar{a}}(\gamma):=\left\{x \in \Lambda|| x-x_{j} \mid \geq \bar{a}, x_{j} \in \gamma, \gamma \in \operatorname{Gamma}_{\Lambda}^{(N)}\right\}
$$

and chose the $\Lambda$ sufficiently large and $\bar{a}$ sufficiently small to satisfy the following inequality:

$$
\sigma\left(\tilde{\Lambda}_{\bar{a}}(\gamma)\right) \geq \frac{1}{2} \sigma(\Lambda)
$$

Then, taking into account that for $x \in \tilde{\Lambda}_{\bar{a}}(\gamma)$

$$
\prod_{\Delta \in \bar{\Delta}_{a, \Lambda}} \chi_{-}^{\Delta}(\gamma \cup\{x\})=\prod_{\Delta \in \bar{\Delta}_{a, \Lambda}} \chi_{-}^{\Delta}(\gamma)
$$

and

$$
W(x ; \gamma)=W_{\bar{a}}(x ; \gamma)=\sum_{y \in \gamma} \phi_{\bar{a}}(|x-y|)
$$

we obtain:

$$
I_{\Lambda}(\gamma):=\int_{\Lambda} \mathrm{d} x \mathrm{e}^{-\beta W(x ; \gamma)} \geq \int_{\tilde{\Lambda}_{\bar{a}}(\gamma)} \mathrm{d} x \mathrm{e}^{-\beta W(x ; \gamma)}=\int_{\tilde{\Lambda}_{\bar{a}}(\gamma)} \mathrm{d} x \mathrm{e}^{-\beta W_{\bar{a}}(x ; \gamma)}
$$

Holder's inequality to (49) with respect to probability measure $\mathrm{d} x / \sigma\left(\tilde{\Lambda}_{\bar{a}}(\gamma)\right)$ gives:

$$
I_{\Lambda}(\gamma) \geq \sigma\left(\tilde{\Lambda}_{\bar{a}}(\gamma)\right) \mathrm{e}^{\frac{-\beta}{\sigma\left(\tilde{\Lambda}_{\bar{a}}(\gamma)\right)^{-}} \int_{\overline{\bar{A}_{\bar{a}}(\gamma)}} W_{\bar{a}}(x ; \gamma) \mathrm{d} x}
$$

Using the property (9) and definition (42) we have:

$$
\int_{\tilde{\Lambda}_{\bar{a}}(\gamma)} W_{\bar{a}}(x ; \gamma) \mathrm{d} x \leq N \int_{\mathbb{R}^{d}}\left|\phi_{\bar{a}}(|x|)\right| \mathrm{d} x:=N\left\|\phi_{\bar{a}}\right\|_{L_{1}}
$$

Using this inequality and taking into account that $N \bar{v} \leq \sigma(\Lambda)$ and (46) we get (43) with

$$
k=\frac{1}{2} \mathrm{e}^{-\frac{2 \beta}{\bar{v}}\left\|\phi_{\bar{a}}\right\|_{L_{1}}}
$$

Taking into account that $Z_{\Lambda \backslash X_{k}}^{(a)}(N-M, \beta)<Z_{\Lambda}^{(a)}(N-M, \beta)$ we have:

$$
\frac{Z_{\Lambda \backslash X_{k}}^{(a)}(N-M, \beta)}{Z_{\Lambda}^{(a)}(N, \beta)} \leq \frac{Z_{\Lambda}^{(a)}(N-1, \beta)}{Z_{\Lambda}^{(a)}(N, \beta)} \cdots \frac{Z_{\Lambda}^{(a)}(N-M, \beta)}{Z_{\Lambda}^{(a)}(N-M+1, \beta)} \leq K^{M}
$$

with $K=1 / k \bar{v}$.

Now, the proof of the Theorem 2 follows from the trivial estimates of the combinatorial sums in (40). Let for simplicity $m=2$ in SSS assumption (12). From the condition $m_{1}+\cdots+m_{k}=M$, one can obtain that $m_{1}^{2}+\cdots+m_{k}^{2} \geq M^{2} / k$. So, we have:

$$
\begin{aligned}
Z_{\Lambda}^{(+)}(N, a, \beta) & \leq 1+\sum_{k=\left\{\left\{\Delta_{1}, \cdots, \Delta_{k}\right\} \subset \bar{\Delta}_{a, \Lambda}\right.}^{N_{\Lambda}}\left(\mathrm{e}^{2 d}\right)^{k} \mathrm{e}^{-4 \beta\left(A(a)-\frac{1}{2} C(a)\right)^{k}} \sum_{M^{\prime}=0}^{\infty} a^{d M^{\prime}} \mathrm{e}^{-4 \beta\left(A(a)-\frac{1}{2} C(a)\right) M^{\prime}} \\
& \leq(1+\epsilon(a))^{N_{\Lambda}}
\end{aligned}
$$

with

$$
\epsilon(a):=2 \mathrm{e}^{2 d} \mathrm{e}^{-4 \beta\left(A(a)-\frac{1}{2} C(a)\right)}
$$


It is clear from the Equations (15), (16), (38) that

$$
\lim _{a \rightarrow 0} \frac{1}{a^{d}} \log (1+\varepsilon(a))=0
$$

so, this gives the proof of the main result. $\square$

\section{Conclusion}

The main result of the article is presented by the Theorem 2.2. It proves that all thermodynamics properties of the infinite system which is defined by phase space (2.1) and interaction potential (2.9) - (2.11) can be described by the cell gas model, phase space and thermodynamics descriptions which are determined by the formulas (2.7), (2.22) - (2.25). In other words, this model approximates the statistical continuous system of interacting point particles up to any preassigned accuracy. It is needed to mark another surprising fact that the set $\Gamma^{\text {(dil) }}$ is subset of measure zero in $\Gamma$ with respect to Poisson measure (see Proposition 3.1 in [4]).

\section{Acknowledgements}

We thank the referee for valuable remarks which improved the original version. The authors gratefully acknowledge the financial support of the Ukrainian Scientific Project "Investigation of the spectral characteristics and critical behavior of complex systems of mathematical physics” (2011-2015).

\section{References}

[1] Huang, K. (1963) Statistical Mechanics. John Wiley and Sons, Inc., London.

[2] Berezin, F.A. and Sinai, Ya.G. (1967) Transactions of the Moscow Mathematical Society, 17, 197-212.

[3] Dobrushin, R.L. (1967) Berk. Sym. Mat. Stat. Prob., VII, 73-87.

[4] Rebenko, A.L. (2013) Reviews in Mathematical Physics, 25, 1-28.

[5] Rebenko, A.L. and Tertychnyi, M.V. (2007) Proc. Inst. Math. NASU, 4, 172-182.

[6] Rebenko, A.L. and Tertychnyi, M.V. (2009) Journal of Mathematical Physics, 50, 0333301-033310. arXiv:0901.0826

[7] Petrenko, S.M., Rebenko, A.L. and Tertychnyi, M.V. (2010) Ukrainian Mathematical Journal, 63, 425-440. arXiv:1007.4325

[8] Albeverio, S., Kondratiev, Yu.G. and Röckner, M. (1998) Journal of Functional Analysis, 154, 444-500. http://dx.doi.org/10.1006/jfan.1997.3183

[9] Rebenko, A.L. (2014) Proc. Inst. Math. NASU, 11, 257-315.

[10] Ruelle, D. (1970) Communications in Mathematical Physics, 18, 127-159. http://dx.doi.org/10.1007/BF01646091

[11] Ruelle, D. (1963) Helvetica Physica Acta, 36, 183-197.

[12] Ruelle, D. (1969) Statistical Mechanics (Rigorous Results). W.A. Benjamin, Inc., Amsterdam.

[13] Rebenko, A.L. and Tertychnyi, M.V. (2008) Methods of Functional Analysis and Topology, 14, 287-296.

[14] Park, Y.M. (1984) Communications in Mathematical Physics, 94, 1-33. http://dx.doi.org/10.1007/BF01212347

[15] Dobrushin, R.L. (1964) Teoriya Veroyatnostei i ee Primeneniya, IX, 626-643.

[16] Dobrushin, R.L. and Minlos, R.A. (1967) Teoriya Veroyatnostei i ee Primeneniya, XII, 595-618. 
Scientific Research Publishing (SCIRP) is one of the largest Open Access journal publishers. It is currently publishing more than 200 open access, online, peer-reviewed journals covering a wide range of academic disciplines. SCIRP serves the worldwide academic communities and contributes to the progress and application of science with its publication.

Other selected journals from SCIRP are listed as below. Submit your manuscript to us via either submit@scirp.org or Online Submission Portal.
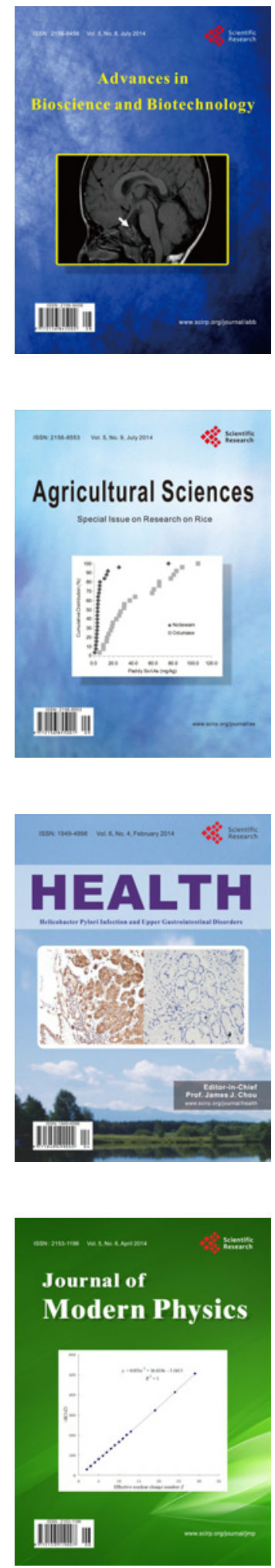
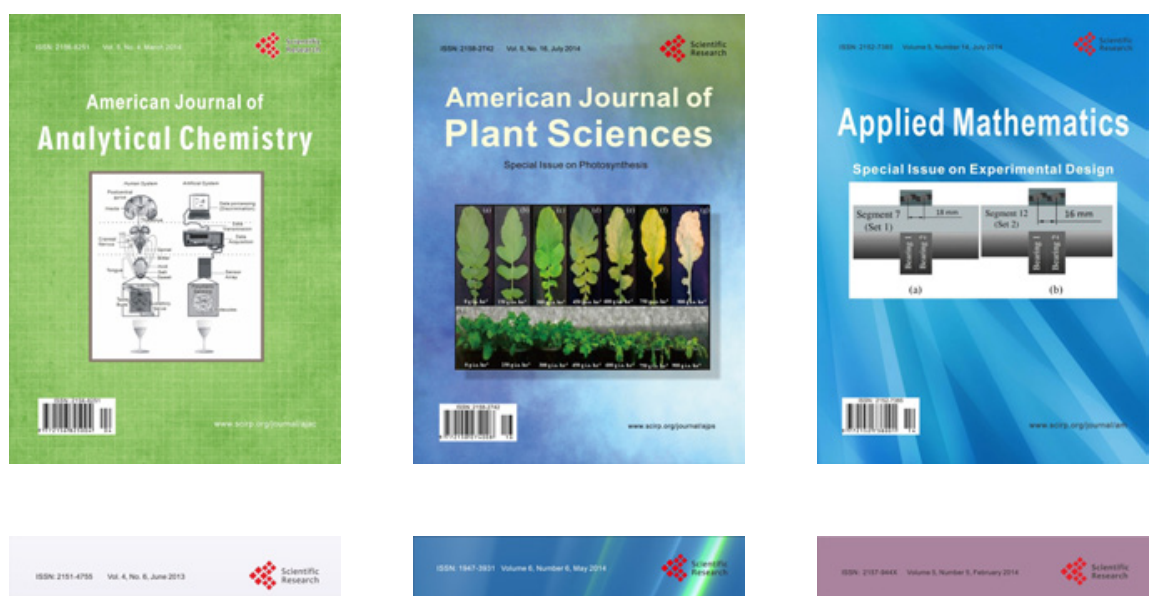

Creative Education
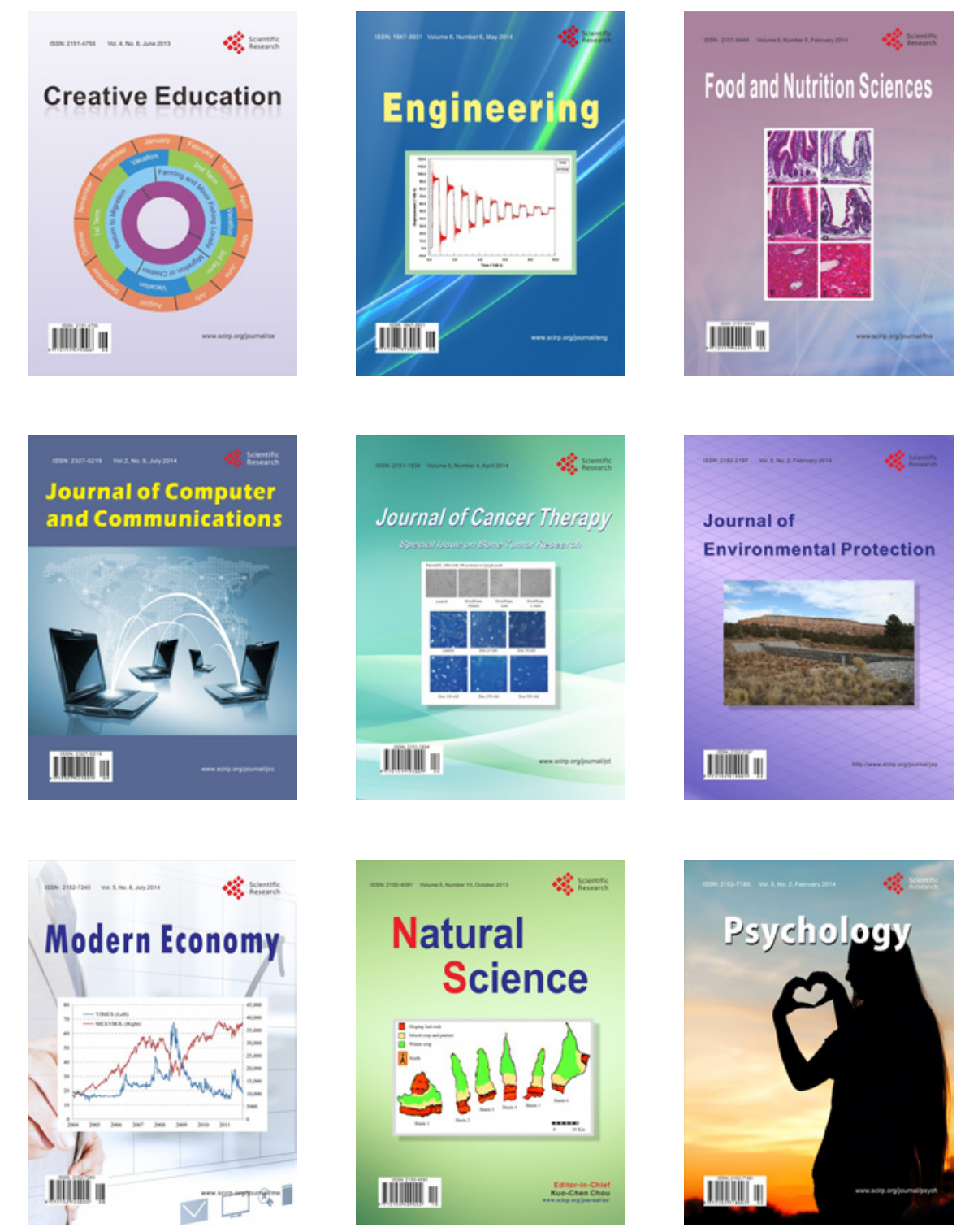